\title{
Single-Port Total Abdominal Colectomy for Colon Cancer
}

\author{
Maen Aboul Hosn, MD, Faek R. Jamali, MD, FSSO, Ramzi Alami, MD \\ Division of General Surgery, Department of Surgery, American University of Beirut, Beirut, Lebanon (all authors).
}

\begin{abstract}
Introduction: Single-incision laparoscopic surgery has gained popularity in recent years with an increasing number of complicated procedures being performed using this approach. Single-port total colectomy has been reported in the literature mainly in the setting of inflammatory bowel disease and polyposis. We report on a young patient who underwent single-port abdominal colectomy for transverse colon cancer along with multiple colonic polyps.

Case Description: A 33-year-old female presented initially with a vague abdominal pain. Her workup included a colonoscopy that showed multiple colonic polyps with a circumferential fungating lesion in the transverse colon. This lesion was biopsied and found to be positive for moderately differentiated adenocarcinoma of the colon. With these findings, the patient was advised about the different surgical options, and the decision was made to go for abdominal colectomy with ileorectal anastomosis. Given this patient's low BMI and favorable body habitus, the procedure was performed with a single-port technique through a small umbilical incision. There were no perioperative complications, and the final pathologic examination revealed a $5-\mathrm{cm}$ invasive moderately differentiated carcinoma extending through the muscularis propria into pericolonic fat and multiple colonic tubulovillous adenomas along with 22 regional lymph nodes draining the basin of the distal transverse colon, all of which were negative for malignancy.
\end{abstract}

Conclusion: Single-port laparoscopic total colectomy for colon cancer in carefully selected patients and in the hands of a fully trained laparoscopic surgeon is feasible and appears to offer more cosmetic benefits than conventional laparoscopic colectomy, with similar short-term and oncological outcomes.

Key Words: Single-port total colectomy, Single-incision surgery, Colon cancer, Laparoscopic total colectomy

Citation Aboul Hosn M, Jamali FR, Alami R. Single-port total abdominal colectomy for colon cancer. CRSLS e2014.00149. DOI: 10.4293/CRSLS.2014.00149.

Copyright (c) 2014 SLS This is an open-access article distributed under the terms of the Creative Commons Attribution-Noncommercial-ShareAlike 3.0 Unported license, which permits unrestricted noncommercial use, distribution, and reproduction in any medium, provided the original author and source are credited.

Address correspondence to: Maen Aboul Hosn, MD, Division of General Surgery, Department of Surgery, American University of Beirut, PO Box 11-0236, Riad El-Solh, Beirut 1107 2020, Lebanon. Telephone: 00961-3-079863, E-mail: ma198@aub.edu.lb

\section{INTRODUCTION}

The single-port approach in general surgery was first reported in 1997 by Navarra et al, ${ }^{1}$ who described the first single-port laparoscopic cholecystectomy. Since then, this approach has been used more frequently, particularly in operations involving a single quadrant of the abdomen such as cholecystectomy, sleeve gastrectomy, and partial colectomy. With the growing laparoscopic experience among surgeons and advent of newer port devices, more complex operations involving all the quadrants, such as total colectomy, have been more frequently attempted. Most reported cases in the literature have involved benign colonic disease. Single-port total colectomy for colonic malignancy has only been reported once, and this reflects the technical difficulties faced and the advanced laparoscopic skills required to perform a complete oncologic resection in such a setting. This case report highlights the approach and techniques used to perform total abdominal colectomy in a young woman with early-stage colon cancer and multiple colonic polyps.

\section{CASE REPORT}

Our patient is a 33-year-old woman with a body mass index of $21 \mathrm{~kg} / \mathrm{m}^{2}$ who presented with a yearlong complaint of recurrent vague abdominal pain and bloating with recent fresh blood per rectum. Her surgical history included open appendectomy at a young age and a C-section delivery. Her family history was significant for breast 


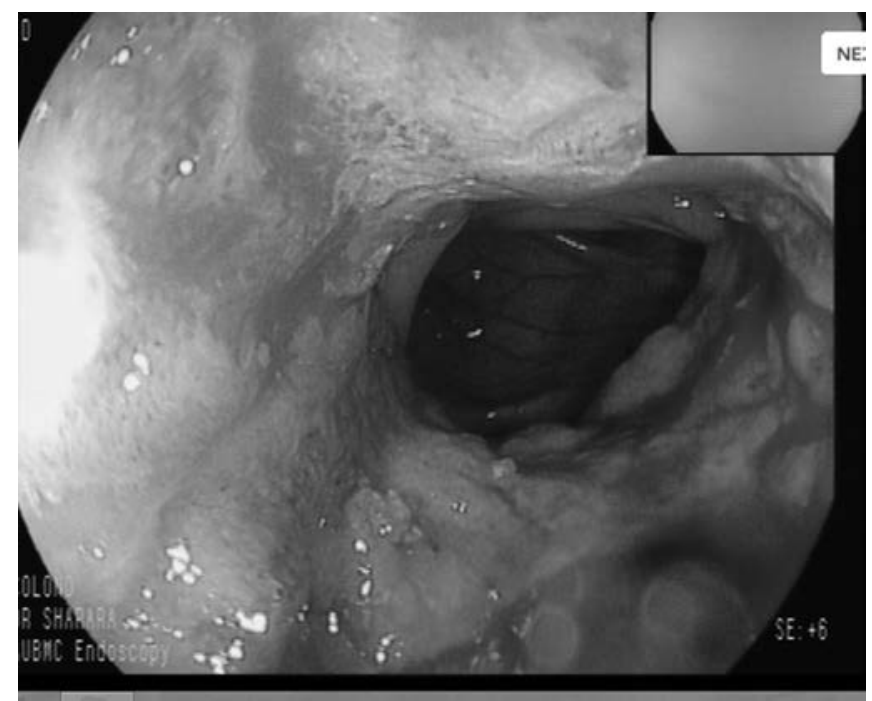

Figure 1. Colonoscopy showing ulcerated mass at distal transverse colon.

cancer in her mother at the age of 55 years. The diagnostic workup included ultrasonography of the abdomen and upper endoscopy, both of which were nonrevealing. Colonoscopy was ultimately performed, showing multiple $(>20)$ large polyps with sizes ranging between 8 and 25 $\mathrm{mm}$ involving the descending and sigmoid colon along with a fungating circumferential ulcerated mass at the distal transverse colon, just proximal to the splenic flexure (Figure 1). Biopsy of the mass yielded positive findings for moderately differentiated adenocarcinoma. On the basis of these findings, the patient was counseled about the high likelihood of harboring a colonic polyposis syndrome and was accordingly advised about the different surgical options, mainly left extended hemicolectomy versus total colectomy with ileorectal anastomosis. The patient elected to undergo total colectomy but had concerns about the postoperative cosmetic results. Given her low body mass index, she was deemed a suitable candidate for the single-port approach.

\section{Surgical Procedure}

With the patient in the lithotomy position, a 4-cm longitudinal incision was made through the umbilicus. The Gelpoint device (Applied Medical, Rancho Santa Margarita, California) was the single-port device used in this procedure. The Alexis portion of the device was inserted through the incision and the cap preloaded with three 10-mm trocars and a 12- $\mathrm{mm}$ trocar (Figure 2). Pneumoperitoneum was then insufflated up to $15 \mathrm{~mm} \mathrm{Hg}$. A long,

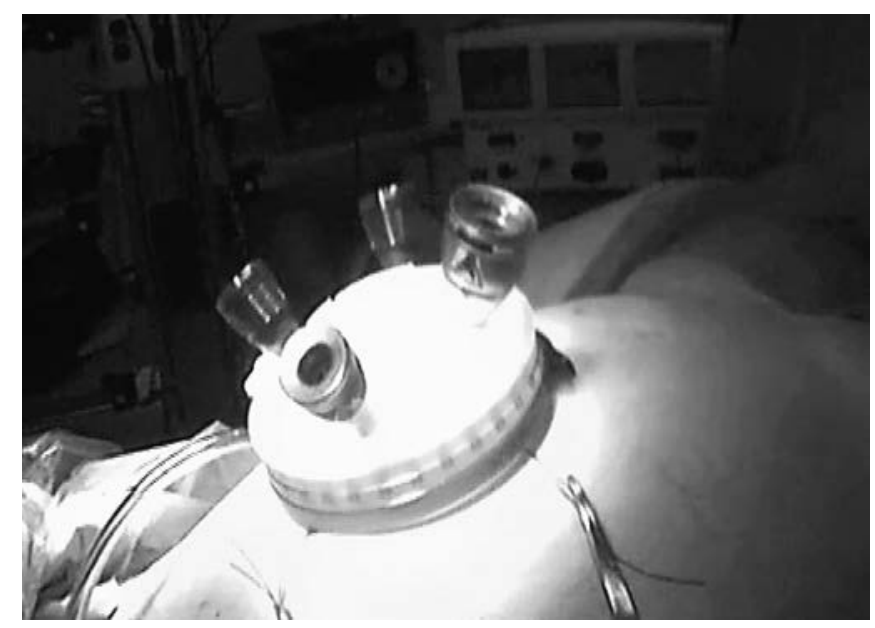

Figure 2. Gelpoint device preloaded with three 10-mm trocars and a $12-\mathrm{mm}$ trocar.

straight, $30^{\circ}$ angled, 8-mm laparoscope was used to avoid cluttering of the instruments outside the body.

On inspection of the peritoneal cavity, there were omental adhesions to the cecum and previous appendectomy site and there was a large $(>5 \mathrm{~cm})$ right adnexal cyst. The liver and peritoneal surface appeared normal with no signs of metastatic disease. The patient was then placed in the Trendelenburg position with the right side up. The surgeon and assistant were at the patient's left side. The camera was placed in the left trocar, and a 5-mm LigaSure device with Autosuture (Valleylab, Boulder, Colorado) was placed in the superior trocar. A medial-to-lateral approach was used for the right colon. Omental adhesions to the colon were taken down and the ileocolic pedicle identified. The ileocolic artery and vein were divided with the LigaSure device and a window opened medially into the retroperitoneum (Figure 3). The retroperitoneal dissection was then carried cephalad until the C-loop of the duodenum and the wall of the transverse colon were easily identified. The mesentery to the right colon was divided at its base, and the areolar attachments between the duodenum and the colon were liberated. The right colon was then mobilized along the white line, starting at the cecum up to the hepatic flexure, which was taken down.

The patient was then placed in a reverse Trendelenburg position for the next portion of the operation, and the laparoscope was placed in the right trocar. The surgeon and assistant were still mostly on the left side but occasionally standing between the patient's legs. The omental attachments to the transverse colon were then mobilized starting from the hepatic and splenic flexures. 


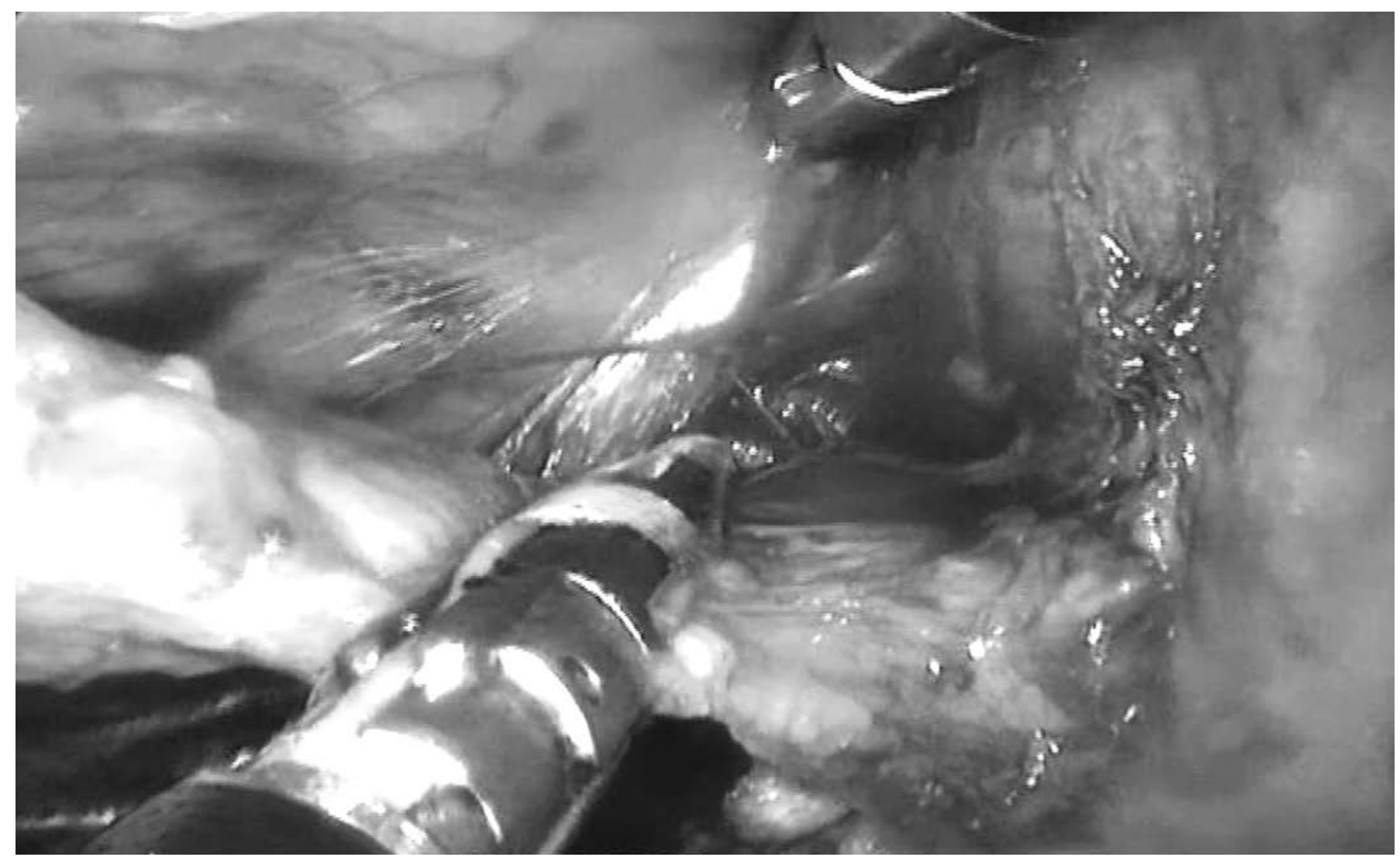

Figure 3. Division of ileocolic artery.

Attention was turned to dissection of the middle colic artery and vein. These were clearly identified with care to protect the superior mesenteric artery, duodenum, and pancreas. The middle colic artery and vein were ligated at their base. Dissection of the left side followed a lateral-tomedial approach. The splenic flexure was fully mobilized, the ligament of Treitz and superior mesenteric vein identified, and the superior mesenteric vein ligated before the patient was placed in the Trendelenburg position again.

Attention was then turned to the identification, dissection, and ligation of the inferior mesenteric artery at its root. The laparoscope was placed in the left trocar and the LigaSure device in the right, and a medial-to-lateral approach was again adopted for the sigmoid mobilization. For this portion and from here on, the patient and assistant were on the patient's right side with the patient placed left side up. The left ureter was identified and protected at this point, and the mesentery of the sigmoid and descending colon was divided with the LigaSure device all the way down and just beyond the peritoneal reflection (Figure 4). The colon was then mobilized off its lateral attachments along the white line of Toldt.
A 60-mm purple Endo GIA (Covidien, New Haven, Connecticut) was deployed to divide the rectosigmoid colon just below the peritoneal reflection. When fully divided, the colon and distal ileum were then delivered through the umbilical incision with the wound protector in place (Figure 5). A small enterotomy was made in the terminal ileum, and the anvil of a 28-mm circular stapler (Covidien) was introduced proximal to the enterotomy. The terminal ileum was divided with another linear 60-mm purple load and the entire abdominal colon handed off as a specimen. The circular anvil was then brought out through the side wall of the distal ileum along the antimesenteric aspect. The distal ileum was dunked back into the peritoneal cavity, and the pneumoperitoneum was re-established. An end-to-end ileorectal anastomosis was then created using the circular stapler, and insufflation of air through a rigid sigmoidoscope showed no air leak.

Given patient's significant family history and high risk of ovarian torsion, the right ovarian cyst was drained and underwent biopsy. The estimated blood loss for the procedure was $50 \mathrm{~mL}$. 
Single-Port Total Abdominal Colectomy for Colon Cancer: A Case Report and Review of Literature, Aboul Hosn M et al.

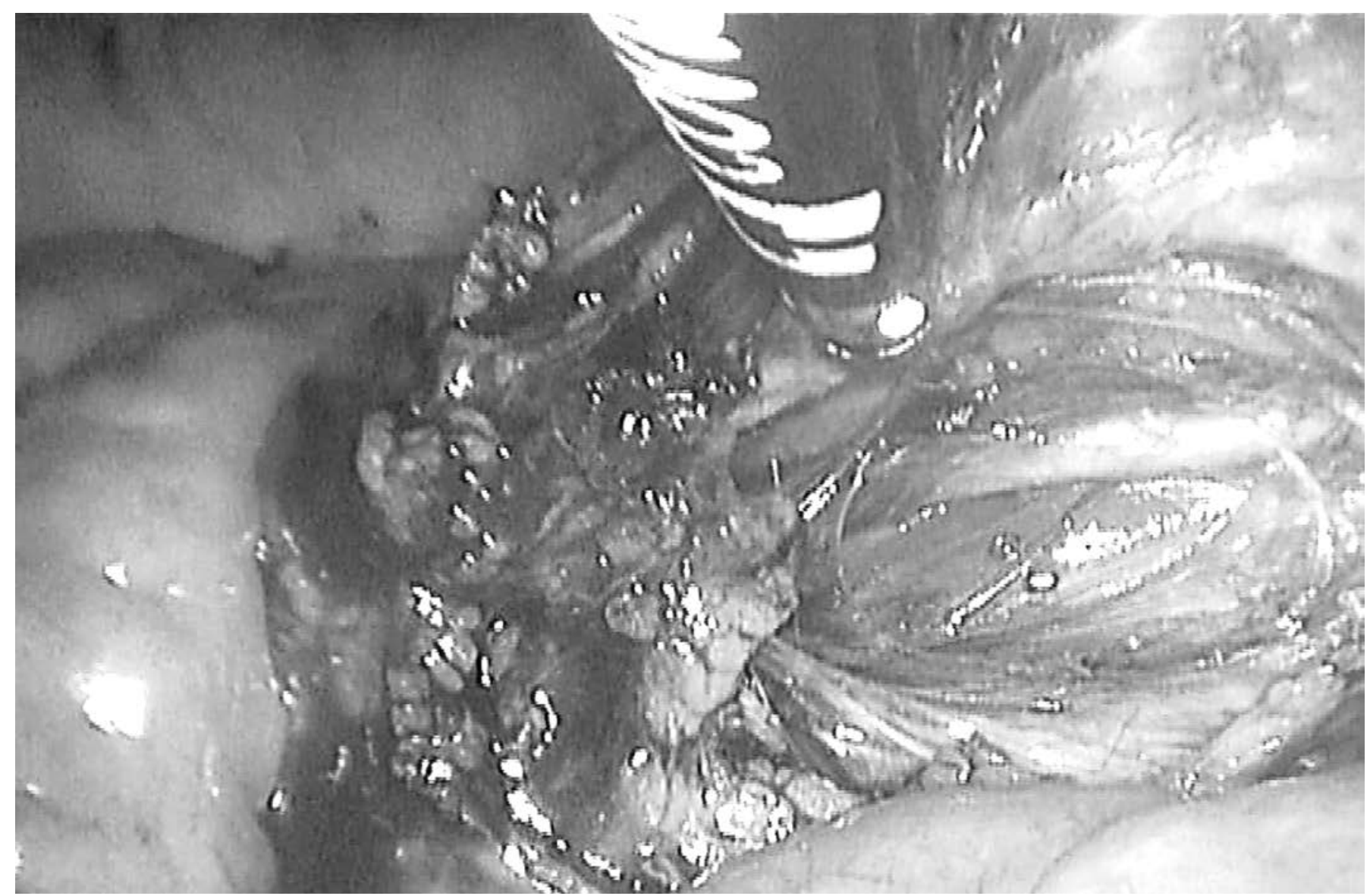

Figure 4. Division of inferior mesenteric artery with left ureter seen in background.



Figure 5. Colon and terminal ileum exteriorized through umbilical incision.

\section{Postoperative Course}

The patient had an uneventful postoperative course and was started on an oral diet 3 days after the operation. She was discharged home on the sixth postoperative day, and



Figure 6. Umbilical incision 6 days after operation. 
her wound was exposed (Figure 6). At the time of discharge, she had recovered bowel function and had diarrhea that was easily manageable at home. The final pathology report showed a $5-\mathrm{cm}$ invasive, moderately differentiated carcinoma extending through the muscularis propria into the pericolonic fat and multiple colonic tubulovillous adenomas, along with 114 total harvested pericolonic lymph nodes and 22 regional lymph nodes draining the basin of the distal transverse colon, all of which yielded negative findings for malignancy. The right ovarian biopsy specimen showed a simple cyst.

\section{DISCUSSION}

The first single-incision surgical procedure was reported in 1997 when Navarra et $\mathrm{al}^{1}$ described a "one-wound" laparoscopic cholecystectomy. However, it was not until 2008 that single-port surgery started gaining momentum, mainly because of improved single-port access techniques and laparoscopic instrumentation, as well as the growing expertise among laparoscopic surgeons. As such, the rate of publication on the topic of single-port laparoscopic surgery has increased drastically over the past 6 years in all surgical disciplines. ${ }^{2}$

\section{Access Technique}

Various different access techniques for single-incision surgery have been described in the literature. Examples include the use of a single skin incision with multiple fascial punctures, wound protectors with surgical gloves, and single-port access devices. ${ }^{3,4}$ Recently, single-port access devices with 3 to 4 operating channels have been more widely used by various surgeons and reported in literature. ${ }^{5}$ The Gelpoint device was the single-port device selected for our case, mainly because its cap provided an additional outer working area facilitating tissue triangulation without the need for angulated instruments. In addition, the rotation of the outer ring of the device around the inner ring allowed for a $360^{\circ}$ adjustment of the trocar configuration as needed. ${ }^{6}$

\section{Technical Challenges}

Single-incision laparoscopic partial colectomy was first described in 2008 by Remzi et $\mathrm{al}^{7}$ and has been performed with increasing frequency since. This procedure, however, entails increased technical difficulty, mainly because of the need to mobilize, retract, and remove a larger specimen, as compared with the more commonly performed single-incision laparoscopic cholecystectomy or appendectomy. ${ }^{8}$ Currently, conventional laparoscopic techniques for partial or total colectomy typically involve either an extraction site or a hand-port site and the use of up to 5 additional trocars. 9 Therefore the theoretical advantages of single-incision laparoscopic colectomy (SILC) compared with multiport laparoscopic colectomy (MLC) include a smaller abdominal incision, potentially reduced postoperative pain, and improved cosmetic result. On the other hand, the main challenge of SILC performed with a conventional rigid laparoscope and straight instruments is the crowding over the access port or access site, leading to clashing of surgical instruments. In addition, the handling of both straight instruments in parallel with the laparoscope through a small single incision decreases the freedom of motion for the surgeon and hinders handling of the laparoscope for the assistant. ${ }^{10}$ Furthermore, the lack of tissue triangulation significantly increases difficulty of colonic exposure and dissection, necessitating maneuvers that are generally avoided in conventional laparoscopic procedures such as crossing of instruments and pushing the specimen away from the camera to achieve proper retraction and exposure. ${ }^{10}$ In attempts to work their way around the problem of triangulation, some surgeons advocate the use of articulated and angulated instruments. ${ }^{11}$ However, the proper use of such instruments has its own learning curve. ${ }^{12}$ In our case, we used the standard laparoscopic instruments. However, we elected to use a long $30^{\circ}$ laparoscope because it improves surgical exposure and helps avoid cluttering of instruments near the port entry site.

\section{Single-Port Partial Colectomy}

Putting the technical difficulties aside, the main question before adopting any new surgical procedure remains whether the approach is safe and feasible and achieves similar oncologic outcomes when compared with the standard of care, which in this case is MLC. In a systematic review of 23 studies with 378 patients undergoing SILC that was published in 2012, it was found that SILC carried relatively low rates of overall mortality $(0.5 \%)$ and morbidity (12.9\%) with operative time and estimated blood loss comparable with published data on MLC. ${ }^{13}$ In addition, the rate of conversion to an open procedure (1.6\%) in these series of SILC was significantly lower when compared with the rate of $10 \%-21 \%$ with MLC. These results, however, did not necessarily translate into a shorter length of stay in SILC cases compared with MLC cases.

\section{SILC for Malignancy}

The use of SILC for colonic malignancy has also been widely studied. A cohort study of 73 patients by McNally 
et $\mathrm{al}^{14}$ in 2011 concluded that SILC can be used safely in selected colon cancer patients with no difference in blood loss, operating room time, or lymph node retrieval. A similar study by Egi et al ${ }^{15}$ comparing surgical outcomes for colon cancer showed no significant differences regarding the perioperative and oncologic results between SILC and MLC. The only reported advantage of SILC was the better cosmetic outcome. ${ }^{15}$ There is only 1 randomized controlled trial in the literature comparing SILC and MLC for colonic neoplasms. ${ }^{16}$ In this study, Poon et al ${ }^{16}$ selected patients with small cancers $(<4 \mathrm{~cm})$ or adenomatous polyps requiring colectomy and randomized them to undergo either SILC or MLC. Patients were blinded to the procedures, and postoperative pain was used as the primary outcome measure. The results showed that SILC significantly reduced postoperative pain and was associated with a shorter hospital stay in good-risk patients with small colonic neoplasms.

The largest meta-analysis comparing SILC with MLC was performed by Zhou et $\mathrm{al}^{17}$ in 2012 and included a total of 14 studies with 1155 patients, of whom 521 underwent SILC and 634 underwent MLC for colorectal disease. The results of their study showed that SILC did not increase the conversion rate and prolong the operative time compared with MLC, with similar operative adverse events. Pooled analysis also showed decreased blood loss for SILC compared with MLC, and this translated into a decreased transfusion requirement rate in the SILC group. There were no differences observed between the 2 groups in terms of tumor margin, length of resected specimens, and harvested lymph nodes, as well as recovery of gastrointestinal function, initiation of oral diet, and overall hospital stay. The main advantage seen in SILC patients was related to the size of the incisions and cosmetic results.

\section{Single-Port Total Colectomy}

Most published data on SILC examine its use in the setting of partial colonic resection. Single-port total colectomy, on the other hand, has been reported in only 50 cases in the literature, most which were performed in the setting of inflammatory bowel disease and familial polyposis syndromes. ${ }^{18-21}$ Only 1 case describing single-port total $\mathrm{CO}^{-}$ lectomy with ileorectal anastomosis for colonic malignancy has been reported. ${ }^{22}$ In that case, access into the abdomen was achieved through a $3-\mathrm{cm}$ longitudinal incision at the midpoint between the umbilicus and the symphysis pubis, and the authors reported no immediate intraoperative or postoperative complications, with the final pathology report showing a $5 \times 4 \times 3-\mathrm{cm}$ adenocarcinoma of the cecum with 17 negative lymph nodes. ${ }^{22}$
Our case report is the second reported single-port total colectomy in the setting of colonic malignancy and the first one performed through an umbilical incision. Despite the fact that laparoscopic lymphadenectomy for distal transverse colon cancer is considered technically challenging with a lower lymph node yield because it involves removing the lymph nodes along the middle colic and ascending branch of the left colonic arteries, ${ }^{23}$ the number of harvested regional lymph nodes in our case (22 regional lymph nodes) was comparable with what has been described in the literature for both SILC and MLC, further validating the use of this approach for oncologic surgical procedures.

\section{CONCLUSION}

Our case shows that single-port laparoscopic total colectomy for colon cancer in the hands of a fully trained laparoscopic surgeon is feasible and appears to offer more cosmetic benefits than conventional laparoscopic colectomy, with similar short-term and oncologic outcomes. It also shows that the use of specialized angulated instruments may not apply to experienced laparoscopic surgeons who can perform the procedure using conventional laparoscopic instruments. A cautious approach, however, with careful patient selection is essential because safe surgical principles should always take priority over the desire for better cosmetic results.

\section{References:}

1. Navarra G, Pozza E, Occhionorelli S, et al. One-wound laparoscopic cholecystectomy. Br J Surg. 1997;84(5):695.

2. Waters JA, Rapp BM, Guzman MJ, et al. Single-port laparoscopic right hemicolectomy: the first 100 resections. Dis Colon Rectum. 2012;55(2):134-139.

3. Huang CK, Lo CH, Houng JY, et al. Surgical results of single-incision transumbilical laparoscopic Roux-en-Y gastric bypass. Surg Obes Relat Dis. 2012;8(2):201-207.

4. Hong TH, You YK, Lee KH. Transumbilical single-port laparoscopic cholecystectomy: scarless cholecystectomy. Surg Endosc. 2009;23(6):1393-1397.

5. Cusati D, Swain JM, Kendrick M, et al. Evaluation of commercially available port access devices for single-incision laparoscopy. Surg Laparosc Endosc Percutan Tech. 2011;21(3): e134-e137.

6. Bucher P, Pugin F, Morel P. Single-port access laparoscopic radical left colectomy in humans. Dis Colon Rectum. 2009; 52(10):1797-1801. 
7. Remzi FH, Kirat HT, Kaouk JH, et al. Single-port laparoscopy in colorectal surgery. Colorectal Dis. 2008;10(8):823-826.

8. Romanelli JR, Earle DB. Single-port laparoscopic surgery: an overview. Surg Endosc. 2009;23(7):1419-1427.

9. Boushey RP, Marcello PW, Martel G, et al. Laparoscopic total colectomy: an evolutionary experience. Dis Colon Rectum. 2007; 50(10):1512-1519.

10. Leblanc F, Champagne BJ, Augestad KM, et al. Single incision laparoscopic colectomy: technical aspects, feasibility, and expected benefits. Diagn Ther Endosc. 2010;2010:913216.

11. Rane A, Kommu S, Eddy B, et al. Clinical evaluation of a novel laparoscopic port (R-Port) and evolution of the single laparoscopic port procedure (SLiPP) J Endourol. 2007;21:A22A23.

12. Xu AA, Zhu JF, Xie X, et al. Mechanical evaluation of articulating instruments and cross-handed manipulation in laparoendoscopic single-site surgery. Surg Innov. In press 2013. Epub 21 Nov 2013.

13. Makino T, Milsom JW, Lee SW. Feasibility and safety of single-incision laparoscopic colectomy: a systematic review. Ann Surg. 2012;255(4):667-676.

14. McNally ME, Todd Moore B, Brown KM. Single-incision laparoscopic colectomy for malignant disease. Surg Endosc. 2011;25(11):3559-3565.

15. Egi H, Hattori M, Hinoi T, et al. Single-port laparoscopic colectomy versus conventional laparoscopic colectomy for colon cancer: a comparison of surgical results. World J Surg Oncol. 2012;24:10:61.
16. Poon JT, Cheung CW, Fan JK, et al. Single-incision versus conventional laparoscopic colectomy for colonic neoplasm: a randomized, controlled trial. Surg Endosc. 2012;26(10):2729-2734.

17. Zhou YM, Wu LP, Zhao YF, et al. Single-incision versus conventional laparoscopy for colorectal disease: a meta-analysis. Dig Dis Sci. 2012;57(8):2103-2112.

18. Paranjape C, Ojo OJ, Carne D, et al. Single-incision laparoscopic total colectomy. JSLS. 2012;16(1):27-32.

19. Chatzizacharias NA, Torrente F, Brennan M, et al. Single port laparoscopic subtotal colectomy and ileostomy in an adolescent with ulcerative colitis. J Crohns Colitis. 2012;6(10):1031-1033.

20. Kanakala V, Borowski DW, Agarwal AK, et al. Comparative study of safety and outcomes of single-port access versus conventional laparoscopic colorectal surgery. Tech Coloproctol. 2012;16(6):423-428

21. Vestweber B, Galetin T, Lammerting K, et al. Single-incision laparoscopic surgery: outcomes from 224 colonic resections performed at a single center using SILS. Surg Endosc. 2013;27(2): $434-442$.

22. Bardakcioglu O, Ahmed S. Single incision laparoscopic total abdominal colectomy with ileorectal anastomosis for synchronous colon cancer. Tech Coloproctol. 2010;14(3):257261.

23. Matsuda T, Fujita H, Kunimoto Y, et al. Clinical outcomes of laparoscopic surgery for transverse and descending colon cancers in a community setting. Asian J Endosc Surg. 2013; 6(3):186-191. 